\title{
Self-Monitoring of Blood Glucose
}

\author{
Satish K. Garg ${ }^{1}$ and Irl B. Hirsch ${ }^{2}$
}

\section{Introduction}

ELF-MONitoring OF BLOOD GLUCOSE (SMBG) is now more than halfway through $\checkmark$ its third decade of use, yet despite its lack of novelty it remains the cornerstone of diabetes therapy. Still, the amount of research regarding its use, application, and the technology itself continues to dwindle. For example, when reviewing the literature for the ATTD 2012 Yearbook the number of articles on SMBG was much more than we have to choose from now. It was quite difficult just a few years ago to pick from the wide choice of articles for this review. Now, while the quality of the articles seemed quite good, the quantity was quite limited.

The themes of the literature for SMBG have not changed. Accuracy remains a major concern, but the traditional name brand meters seem to do quite well. Alternate site testing, use of SMBG for telemedicine, inpatient SMBG, and the use of SMBG in the pediatric age group continues to be addressed, although not in the volume we saw 10 years ago. We hope you find this review of the past year in SMBG helpful.

\section{Key Articles Reviewed for this Article}

Perioperative glucose monitoring and treatment of patients undergoing vascular surgery in a community hospital setting

Morrison $S^{1}$, O'Donnell $J^{2}$, Ren $D^{3}$, Henker $R^{4}$

AANA J 2014; 82: 427-30

Can gingival crevicular blood be relied upon for assessment of blood glucose level?

Dwivedi $S^{1}$, Verma $S J^{2}$, Shah $M^{3}$, Jain $K^{1}$

N Y State Dent J 2014; 80: $38-42$

Internet blood glucose monitoring systems provide lasting glycemic benefit in type 1 and 2 diabetes: a systematic review

Tildesley $H D^{1}$, Po $M D^{2}$, Ross $S A^{3}$

Med Clin North Am 2015; 99: 17-33

Perceptions and experiences of using automated bolus advisors amongst people with type 1 diabetes: a longitudinal qualitative investigation

Lawton $J^{1}$, Kirkham $J^{1}$, Rankin $D^{1}$, Barnard $K^{2}$, Cooper $C L^{3}$, Taylor $C^{4}$, Heller $S^{5}$, Elliott $J^{5}$, the REPOSE Group

Diabetes Res Clin Pract 2014; 106: 443-50

\footnotetext{
${ }^{1}$ University of Colorado Denver, CO.

${ }^{2}$ University of Washington Medical Center, Seattle, WA.
} 


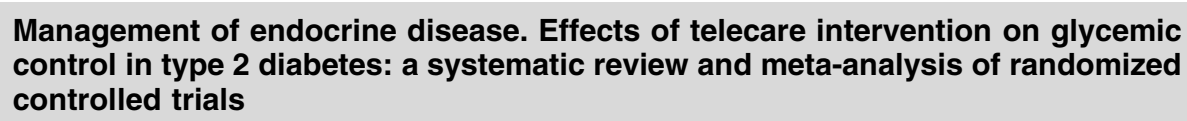

Pettus $J^{1}$, Stenger $P^{2}$, Schachner $H^{3}$, Dunne $N^{3}$, Parkes $J^{3}$, Pardo $S^{3}$, Edelman $S V^{1,4}$ Curr Med Res Opin 2014; 30: 1795-802

Accuracy evaluation of blood glucose monitoring systems in children on overnight closed-loop control

DeSalvo DJ, Shanmugham S, Ly TT, Wilson DM, Buckingham BA

J Diabetes Sci Technol 2014; 8: 969-73

Frequency of mealtime insulin bolus predicts glycated hemoglobin in youths with type 1 diabetes

Patton $S R^{1}$, DeLurgio $S A^{2}$, Fridlington $A^{3}$, Cohoon $C^{3}$, Turpin $A L^{3}$, Clements $M A^{3}$

Diabetes Technol Ther 2014; 16: 519-23

Deciding between using the first or second drop of blood for the self-monitoring of blood glucose

Li M, Wang $X$, Shan Z

Prim Care Diabetes 2014; 8: 365-69

System accuracy evaluation of systems for point-of-care testing of blood glucose: a comparison of a patient-use system with six professional-use systems

Freckmann $G^{l}$, Schmid $C^{l}$, Pleus $S^{l}$, Baumstark $A^{l}$, Link $M^{I}$, Stolberg $E^{l}$, Haug $C^{l}$, Sieber $J^{2}$

Clin Chem Lab Med 2014; 52: 1079-86

\section{Perioperative glucose monitoring and treatment of patients undergoing vascular surgery in a community hospital setting}

Morrison $S^{1}$, O'Donnell $J^{2}$, Ren $D^{3}$, Henker $R^{4}$

${ }^{I}$ Department of Nurse Anesthesia, University of Pittsburgh and North Star Anesthesia, Sewickley, PA; ${ }^{2}$ Department of Nurse Anesthesia, University of Pittsburgh, Pittsburgh, PA; ${ }^{3}$ School of Nursing, University of Pittsburgh, Pittsburgh, PA; ${ }^{4}$ Center for Research and Evaluation, School of Nursing, University of Pittsburgh, Pittsburgh, PA

\section{AANA J 2014; 82: 427-30}

Studies in the past have shown that the glucose control of patients that underwent cardiovascular surgery directly affected the outcome of the surgery: they stayed longer at the hospital, with incidence of infection, stroke, renal failure, and myocardial infarction. Some were readmitted to the hospital within 30 days of their discharge. This article discusses the glucose monitoring and treatment practices of a small community hospital, when its goal is to determine how these practices relate to postoperative complications in patients undergoing vascular surgery. This was a retrospective analysis of 101 patients who underwent vascular surgery and whose charts were reviewed. Details of perioperative blood glucose levels, frequency of monitoring, treatment practices, and postoperative complications were collected from their electronic and paper medical records. Twenty-seven had a blood glucose level greater than $140 \mathrm{mg} / \mathrm{dL}$ preoperatively. Intraoperative blood glucose levels were monitored for eight patients. Nine patients had blood glucose levels treated during the perioperative period. The threshold for treatment of preoperative hyperglycemia was a blood glucose level of $236 \mathrm{mg} / \mathrm{dL}$. Increased attention of perioperative blood glucose levels is needed so that appropriate interventions can be established for the improvement of outcomes. Glucometers must be available to anesthesia providers in order to perform intraoperative monitoring of blood glucose levels.

\section{Comment}

The debate on intensive versus less intensive diabetes management during the perioperative period continues. The investigators treated preoperative hyperglycemia at a level of $236 \mathrm{mg} / \mathrm{dL}$. There is no justification for using such a high number for initiating treatment for hyperglycemia. However, the authors emphasize an important point that glucose meters must be readily available to anesthesiologists so that appropriate peri- and intra- 
operative monitoring of blood glucose can be performed. The major reason for ongoing controversy revolves around the risk of hypoglycemia in this high-risk population in the hospital setting. In addition, a majority of the hospitals still do not have standardized protocols to manage hyperglycemia in the perioperative setting.

\section{Can gingival crevicular blood be relied upon for assessment of blood glucose level?}

Dwivedi $S^{1}$, Verma $S J^{2}$, Shah $M^{3}$, Jain $K^{1}$

${ }^{1}$ Peoples Dental College and Hospital, Bhopal (M.P.) India; ${ }^{2}$ Private Practice, Vadodara (Gujarat) India; ${ }^{3}$ K.M. Shah Dental College, Vadodara (Gujarat) India

\section{N Y State Dent J 2014; 80: 38-42}

Approximately half of the patients suffering from diabetes mellitus (DM) go undiagnosed. In addition, the prevalence of $\mathrm{DM}$ is more than twice as high as in patients with periodontitis when compared to periodontally healthy subjects. Therefore, a high percentage of patients with periodontitis may have undiagnosed DM. The aim of the present study was to determine whether the blood from a gingival crevice during a routine periodontal examination can indicate glucose levels.

\section{Material and Methods}

In total, 75 patients (43 males and 32 females) with chronic periodontitis were divided into two groups - Group I and Group II, respectively-for observational cross-sectional studies. Blood oozing from the gingival crevices of anterior teeth following periodontal probing was collected with the stick of a glucose self-monitoring device, and the blood glucose levels were measured. At the same time, finger-prick blood was taken for glucometric analysis and subsequent readings were recorded.

\section{Results}

The patient's blood glucose values ranged from 74 to $256 \mathrm{mg} / \mathrm{dL}$. The comparison between gingival crevicular blood and finger-prick blood showed a very strong correlation, with a t-value of 3.97 (at $P$-value $=0.001$ ).

\section{Conclusions}

The data from this study have shown that gingival crevicular blood (GCB) collected during diagnostic periodontal examination can be an excellent source of blood for glucometric analysis.

\section{Comment}

Alternate sources of blood continue to be of interest for glucose monitoring in high-risk patients. This study performed in patients with periodontitis found a good correlation of GCB with finger-prick blood glucose values. More importantly, this study highlighted undiagnosed diabetes in patients with periodontitis. The blood glucose measurements correlated well across different glucose ranges from 74 to $256 \mathrm{mg} / \mathrm{dL}$. It is unknown if the rate of change of blood glucose values will be affected at the lower and the higher glucose level since those levels are not available in this study.

\section{Internet blood glucose monitoring systems provide lasting glycemic benefit in type 1 and 2 diabetes: a systematic review}

Tildesley $H D^{1}$, Po $M D^{2}$, Ross $S A^{3}$

${ }^{1}$ Department of Endocrinology and Metabolism, St Paul's Hospital, University of British Columbia, Vancouver, British Columbia, Canada; ${ }^{2}$ SCRIPT, Toronto, Canada; ${ }^{3}$ University of Calgary, Calgary, Canada

Med Clin North Am 2015; 99: 17-33

Internet blood glucose monitoring systems (IBGMS) have been shown to improve glycemic control in patients with type 2 diabetes (T2D) who are pharmacologically managed, using oral agents or insulin. IBGMS improves glycemic levels in patients with type 1 diabetes (T1D) and does not lead to increased hypoglycemia. Mechanisms underlying IBGMSassociated glycemic improvement go beyond optimizing insulin dose titration. The most relevant effects have to do with increased self-motivation of patients and improved communication between patients and physicians. IBGMS have been recommended in clinical practice guidelines, and their effectiveness and safety in trials suggest that this approach is appropriate for patients with T1D or T2D.

\section{Comment}

This study highlights the role of IBGMS. The study documented improved glucose control in patients with type 2 diabetes managed either with oral drugs or insulin treatment. The most important lesson from the study is improved patients' self-motivation and better patient/physician communication. It has been highlighted in several studies that patient engagement and access to the provider results in better outcomes. Electronic communication makes such interactions easier for patients and providers. Longer studies for patients with type 1 and type 2 diabetes will need to be performed before concluding effectiveness of IBGMS.

\section{Perceptions and experiences of using automated bolus advisors amongst people with type 1 diabetes: a longitudinal qualitative investigation}

Lawton $J^{1}$, Kirkham $J^{1}$, Rankin $D^{1}$, Barnard $K^{2}$, Cooper $\mathrm{CL}^{3}$, Taylor $\mathrm{C}^{4}$, Heller $\mathrm{S}^{5}$, Elliott $\mathrm{J}^{5}$, the REPOSE Group

${ }^{1}$ Centre for Population Health Sciences, University of Edinburgh, Edinburgh, UK; ${ }^{2}$ Human Development and Health, Faculty of Medicine, University of Southampton, Southampton, UK; ${ }^{3}$ Clinical Trials Research Unit, University of Sheffield, Sheffield, UK; ${ }^{4}$ The Sheffield Diabetes and Endocrine Centre, Northern General Hospital, Sheffield, UK; ${ }^{5}$ Academic Unit of Diabetes, Endocrinology and Metabolism, University of Sheffield, Sheffield, UK

Diabetes Res Clin Pract 2014; 106: 443-50 
Aims

To explore people's reasons for using bolus advisors to determine insulin doses, as well as their experiences using bolus advisors and their likes/dislikes of this technology.

\section{Subjects and Methods}

A total of 42 people with type 1 diabetes who had received instructions on using bolus advisors during a structured education course were interviewed post-course and 6 months later. Data were analyzed thematically.

\section{Results}

Participants who considered themselves to have poor mathematical skills showed a gratitude for, and heavy reliance on, advisors. Others liked and chose to use advisors because they saved time and effort calculating doses and/or had a data storage facility. Follow-up interviews reflected that, by virtue of no longer calculating their doses, participants could become deskilled and increasingly dependent on advisors. Some forgot what their mealtime ratios were; others reported a misperception that, because they were preprogrammed during courses, the parameters never needed changing. Effective review of blood glucose data could be hindered by the use of data storage facilities, and some participants reported an adverse impact on glycemic control.

\section{Discussion}

While participants liked advisors and perceived benefits to using them, there may be unintended consequences to giving people access to this technology. To promote effective use, ongoing input and education from trained health professionals might be warranted.

\section{Comment}

Patients using MDI or insulin pump increasingly use bolus advisors. Many times bolus advisors take into account insulin on board and calculate the appropriate bolus insulin dose for the meal based on carbohydrate intake. The main point highlighted in this study is patient education by the health provider team to avoid unintended consequences. In addition, it's important that patients do not expect more than what the advisor can provide, as some patients reported adverse effect on their glucose control.

\section{Management of endocrine disease. Effects of telecare intervention on glycemic control in type 2 diabetes: a systematic review and meta-analysis of randomized controlled trials}

\author{
Zhenru Huang, Hong Tao, Qingdong Meng, \\ and Long Jing ${ }^{I}$
}

Department of Endocrinology and Metabolism, Beijing Anzhen Hospital, Capital Medical University, Beijing Institute of Heart Lung and Blood Vessel Diseases, 2 Anzhen Road, Chaoyang District, Beijing 100029, China and ${ }^{I}$ Beijing Anzhen Hospital, Capital Medical University, The Key Laboratory of Remodeling-Related Cardiovascular Diseases, Ministry of Education, Beijing Institute of Heart
Lung and Blood Vessel Diseases, 2 Anzhen Road, Chaoyang District, Beijing 100029, China

Eur J Endocrinol 2015; 172: R93-101

\section{Objective}

To review the published literature on the effects of telecare intervention in patients with type 2 diabetes as well as inadequate glycemic control.

\section{Design and Methods}

From December 8-16, 2013, we conducted a review of randomized controlled trials on telecare intervention in patients with type 2 diabetes, along with a search of electronic databases such as The Cochrane Library, PubMed, EBSCO, CINAHL, Science Direct, Journal of Telemedicine and Telecare, and China National Knowledge Infrastructure (CNKI). The eligible studies were independently selected and reviewed by two evaluators. The following elements were analyzed: changes in HbA1c, fasting plasma glucose (FPG), post-prandial plasma glucose (PPG), body mass index (BMI), and body weight.

\section{Results}

The analysis included 18 studies and 3,798 subjects. It showed that telecare greatly improved the management of diabetes. Mean $\mathrm{HbA1c}$ values were reduced by -0.54 (95\% CI, -0.75 to $-0.34 ; \mathrm{P}<0.05)$, mean FPG levels by $-9.00 \mathrm{mg} /$ $\mathrm{dL}(95 \% \mathrm{CI},-17.36$ to $-0.64 ; \mathrm{P}=0.03)$, and mean PPG levels reduced by $-52.86 \mathrm{mg} / \mathrm{dL}(95 \% \mathrm{CI},-77.13$ to -28.58 ; $\mathrm{P}<0.05$ ) when compared with the group receiving standard care. Meta-regression and subgroup analyses indicated that study location, sample size, and treatment-monitoring techniques were the sources of heterogeneity.

\section{Conclusions}

Patients monitored by telecare showed significant improvement in glycemic control in type 2 diabetes when compared with those monitored by routine follow-up. Significant reduction in HbA1c levels was associated with Asian populations, small sample size, and telecare, and with those patients with baseline HbA1c greater than $8.0 \%$.

\section{Comment}

The global increase in the prevalence of diabetes is likely to affect more than half a billion people over the next decade, especially in countries with limited resources. In addition, lack of providers in the field of diabetes mandates development of telecommunication intervention for management of diabetes. This is a meta-analysis of 18 different studies (Asian population) that concluded that significant improvement in A1c $(-0.54 \%)$ with significant improvement in fasting and postprandial blood glucose levels when compared with the standard care. Telecare also allows reach out to remote populations where limited care is available. It is important that payers should recognize telecare as an alternate way to deliver a similar level of diabetes care as seen in routine clinical practice. Many parts of the world, Telecare is not reimbursed at the same level or at all. 


\section{Testing versus guessing blood glucose values: impact on self-care behaviors in type 2 diabetes}

Pettus $J^{1}$, Stenger $P^{2}$, Schachner $H^{3}$, Dunne $N^{3}$, Parkes $\mathrm{JL}^{3}$, Pardo $\mathrm{S}^{3}$, Edelman $\mathrm{SV}^{1,4}$,

${ }^{1}$ Division of Endocrinology, Diabetes \& Metabolism, University of California at San Diego, San Diego, CA; ${ }^{2}$ Medical Communications, Bayer HealthCare LLC, Diabetes Care, Whippany, NJ; ${ }^{3}$ Department of Medical and Clinical Affairs, Bayer HealthCare LLC, Diabetes Care, Whippany, $\mathrm{NJ} ;{ }^{4}$ Taking Control of Your Diabetes (TCOYD), Del Mar, $C A$

Curr Med Res Opin 2014; 30: 1795-802

\section{Objective}

To assess differences between estimated blood glucose values and those measured on a blood glucose meter and the impact on self-care behavior in type 2 diabetes.

\section{Design and Methods}

Subjects $\geq 18$ years with type 2 diabetes $(\mathrm{N}=297)$ attending a Taking Control of Your Diabetes conference were asked questions about diabetes management and to estimate their current blood glucose. Study staff tested subjects' blood glucose on a meter. After seeing the result, subjects were again asked questions on diabetes management.

\section{Main outcome measure}

The percentage of subject blood glucose estimations that were outside ISO 15197:2003 accuracy criteria ( $> \pm 15 \mathrm{mg} /$ $\mathrm{dL}$ or $> \pm 20 \%$ of meter glucose values).

\section{Results}

Nearly half $(46 \%)$ of subjects estimated blood glucose values outside ISO 15197:2003 accuracy criteria. Time since last blood glucose test, time since last meal, testing frequency, and $\mathrm{A} 1 \mathrm{C}$ did not have an effect on differences between estimated blood glucose values and meter results. In the questionnaire before blood glucose testing, most subjects strongly agreed, agreed, or neither agreed nor disagreed that "I make decisions about my diabetes, such as my food intake or my insulin dose even when I do not test my blood sugar" (71\%) and "My body tells me without testing if my blood sugar is low or high" (77\%). After blood glucose testing, 99\% of subjects strongly agreed, agreed, or neither agreed nor disagreed that "Knowing my blood sugar by checking could help me make different diabetes decisions."

\section{Conclusions}

Self-monitoring of blood glucose is an important component of diabetes self-management. Testing rather than guessing blood glucose values is important to obtain accurate results and inform people with type 2 diabetes to make effective, appropriate diabetes management decisions. A potential limitation of this study is that the subject population may not be representative of the general population of people with diabetes; however, the conference setting may attract a more motivated population, which could underestimate the magnitude of the results.

\section{Comment}

A common defense for many patients why they don't measure blood glucose is they think they can "feel" their glucose levels without testing. This is clearly not the case. While the authors acknowledge the limitations of the study population, my experience is these findings would be true for most patients.

\section{Accuracy evaluation of blood glucose monitoring systems in children on overnight closed-loop control}

\author{
DeSalvo DJ, Shanmugham S, Ly TT, Wilson DM, \\ Buckingham BA
}

\section{Stanford University School of Medicine, Stanford, CA}

J Diabetes Sci Technol 2014; 8: 969-73

In this pilot study, children with type 1 diabetes participating in overnight closed-loop studies were evaluated to determine the difference in accuracy between the Bayer Contour $^{\circledR}$ Next $(\mathrm{CN})$ and HemoCue ${ }^{\circledR}$ (HC) glucose monitoring systems. A total of 28 subjects aged 10-18 years old were admitted to a clinical research center and glucose values were obtained every 30 minutes overnight. Using whole blood samples for $\mathrm{CN}$ and $\mathrm{HC}$ readings, glucose values were measured and results were compared to Yellow Springs Instrument (YSI) reference values obtained with plasma from the same sample. System accuracy was compared using mean absolute relative difference (MARD) and International Organization for Standardization (ISO) accuracy standards. Glucose measurements were evaluated at 457 time points. $\mathrm{CN}$ performed better than $\mathrm{HC}$ with an average MARD of $3.13 \%$ compared to $10.73 \%$ for $\mathrm{HC}(\mathrm{P}<0.001)$. With a limited sample size, CN met ISO criteria (2003 and 2013) at all glucose ranges while $\mathrm{HC}$ did not. $\mathrm{CN}$ performed very well, and would make an excellent meter for future closed-loop studies outside of a research center.

\section{Comment}

One unexpected benefit from the closed-loop studies has been the ability to analyze accuracy of both continuous glucose meters and home blood glucose monitors. This study is surprising, despite the relatively few patients, in that the home meter has a lower MARD than the HemoCue ${ }^{\circledR}$, which has traditionally been a gold standard for home studies. This further supports the improved technology for today's home glucose meters, and makes us wonder why we need to continue to use the more inaccurate meters in the United States for Medicare and indigent patients.

\section{Frequency of mealtime insulin bolus predicts glycated hemoglobin in youths with type 1 diabetes}

Patton $\mathrm{SR}^{1}$, DeLurgio $\mathrm{SA}^{2}$, Fridlington $\mathrm{A}^{3}$, Cohoon $\mathrm{C}^{3}$, Turpin $A L^{3}$, Clements $M A^{3}$

${ }^{1}$ Department of Pediatrics, University of Kansas Medical Center, Kansas City, KS; ${ }^{2}$ Health Outcomes Research 
Department; ${ }^{3}$ Division of Endocrinology, Children's Mercy Hospital, Kansas City, MO

Diabetes Technol Ther 2014; 16: 519-23

\section{Background}

Two electronic measures of adherence exist within pediatric diabetes management: frequency of daily blood glucose monitoring (BGM) and the BOLUS score, a measure of frequency of mealtime insulin bolusing. Previous research has shown the BOLUS score to be superior to daily BGM in predicting youths' glycated hemoglobin $(\mathrm{HbAlc})$ in a cross-sectional study. Using a prospective, longitudinal design, we present data comparing the two adherence measures in predicting $\mathrm{HbAlc}$.

\section{Subjects and Methods}

Blood glucose meter data and insulin pump records were collected from a clinical database of 175 youths with type 1 diabetes (mean age, 11.7 \pm 3.6 years at baseline), as were the youths' $\mathrm{HbA1c}$ levels occurring at the download time and at 3,6 , 9 , and 12 months post-downloads. Using a standardized protocol, the youths' mean BGM and BOLUS score were calculated.

\section{Results}

Intraclass correlations (ICCs) revealed significant absolute equivalence among youths' predicted $\mathrm{HbA1c}$ values using $\mathrm{BO}$ LUS and BGM scores and future actual $\mathrm{HbA} 1 \mathrm{c}$ values up to 12 months post-download. However, the ICCs of BOLUS scores with future $\mathrm{HbAlc}$ values were consistently higher than those of the BGM scores. Also, the predictions of the BOLUS scores were significantly more accurate $(\mathrm{P} \leq 0.002)$ than those of the BGM scores based on the root-mean-squared error of predictions.

\section{Conclusions}

In a prospective, longitudinal design, youths' BOLUS scores were superior to youths' daily BGM in predicting future values of HbA1c. Calculating a BOLUS score versus BGM can help researchers and clinicians better predict the youths' HbAlc.

\section{Comment}

It is well-appreciated that many pediatric patients miss their boluses for both meals and snacks, and this has been a major challenge for modern-day insulin regimens in this age group. It is not surprising that quantitatively a "BOLUS SCORE" is more important to the HbAlc level than that frequency of blood glucose testing. One has to wonder if this is even more of an issue for multiple-daily injection patients where prandial insulin is not as convenient.

\section{Deciding between using the first or second drop of blood for the self-monitoring of blood glucose}

\section{Li M, Wang $X$, Shan $Z$}

Department of Endocrinology and Metabolism, Institute of Endocrinology, Liaoning Provincial Key Laboratory of Endocrine Diseases, The First Affiliated Hospital of China Medical University, Shenyang, PR China

Prim Care Diabetes 2014; 8: 365-69

\section{Aims}

To evaluate whether the first or the second drop of blood is more effective for the self-monitoring of blood glucose (SMBG).

\section{Methods}

Hospitalized patients carried out SMBG using their first and second drop of blood; meanwhile, venous blood glucose was measured. The differences in blood glucose measurements were then compared in groups with varying regions of blood glucose levels. There were 802 groups of blood glucose in 526 patients.

\section{Results}

No significant difference was found in the blood glucose levels of the first and second drop of blood and venous blood. However, after combining then dividing measurements into six groups according to blood glucose concentration, we found statistically significant differences in the groups containing blood glucose values $<9.9$ or $20-30 \mathrm{mmol} / \mathrm{L}$ among the blood glucose levels obtained from the first drop, second drop, and venous blood. Yet, there were no significant differences in the $10-14.9$ or $15-19.9 \mathrm{mmol} / \mathrm{L}$ groups.

\section{Conclusions}

Both the first or second drop of blood can be used for performing SMBG to assess real-time venous glucose in the clinical setting. By categorizing blood glucose into various levels more accurately, we observed that there was no significant difference between the first or second drop of blood and the venous blood glucose value when blood glucose levels were maintained between 10 and $20 \mathrm{mmol} / \mathrm{L}$. In addition, when blood glucose levels were below $10 \mathrm{mmol} / \mathrm{L}$, the value obtained from first drop of blood was close to that from venous blood, whereas when the blood glucose level is $>20 \mathrm{mmol} / \mathrm{L}$, the blood glucose value from the second drop of blood was more accurate.

\section{Comment}

This study brings back memories of the "first-versussecond voided urines" from the urine glucose testing days. This study needs to be repeated with different blood glucose meters to confirm the results. From a practical point of view, both at home and in the hospital it seems most use the first drop of blood, but even this isn't well documented. Assuming the first drop is indeed used most of the time, the greatest impact of this would be for hyperglycemia above $20 \mathrm{mmol} / \mathrm{L}$, for which clinically one could argue accuracy is not as critical with insulin dosing as with hypoglycemia.

\section{System accuracy evaluation of systems for point-of-care testing of blood glucose: a comparison of a patient-use system with six professional-use systems}

Freckmann $G^{l}$, Schmid $C^{l}$, Pleus $S^{l}$, Baumstark $A^{l}$, Link $M^{1}$, Stolberg $E^{1}$, Haug $C^{l}$, Sieber $J^{2}$ 
${ }^{I}$ Institut für Diabetes-Technologie Forschungs- und Entwicklungsgesellschaft mbH an der Universität Ulm, Ulm, Germany; ${ }^{2}$ Sanofi-Aventis Deutschland GmbH, Frankfurt, Germany

Clin Chem Lab Med 2014; 52: 1079-86

\section{Background}

Point-of-care testing (POCT) of blood glucose (BG) is performed by medical personnel in clinical settings as well as by patients at home employing SMBG. Our research aimed to determine if a system mainly intended for SMBG by people with diabetes, but also suitable for BG measurements by medical personnel, can achieve measurement accuracy on capillary blood samples that are comparable to professionaluse POCT systems.

\section{Methods}

System accuracy was evaluated under standardized conditions, following the ISO standard 15197:2003. For each system (which included one SMBG system with three test strip lots and six professional-use systems), measurement results from capillary blood samples of 100 subjects were compared with a standardized laboratory glucose oxidase method (YSI 2300 glucose analyzer).

\section{Results}

The seven evaluated systems showed $99.5 \%$ or $100 \%$ of the measurement results within the required system accuracy limits of ISO $15197: 2003( \pm 0.83 \mathrm{mmol} / \mathrm{L}$ at $\mathrm{BG}$ concentrations $<4.2 \mathrm{mmol} / \mathrm{L}$ and $\pm 20 \%$ at BG concentrations $\geq 4.2 \mathrm{mmol} / \mathrm{L}$ ). Using the more stringent requirements of the revision ISO
15197:2013, the systems showed between $99 \%$ and $100 \%$ of the measurement results within the accuracy limits $( \pm 0.83 \mathrm{mmol} / \mathrm{L}$ at $\mathrm{BG}$ concentrations $<5.55 \mathrm{mmol} / \mathrm{L}$ and $\pm 15 \%$ at $\mathrm{BG}$ concentrations $\geq 5.55 \mathrm{mmol} / \mathrm{L}$ ). In addition, the systems showed between $82 \%$ and $98 \%$ when even more restrictive limits were used $( \pm 0.56 \mathrm{mmol} / \mathrm{L}$ and $\pm 10 \%$, respectively).

\section{Conclusions}

Data from this study, which aimed to determine system accuracy, suggest that SMBG systems can achieve system accuracy that is comparable with professional-use systems when measurements are carried out on capillary blood samples by trained personnel in a standardized and controlled setting.

\section{Comment}

While this is one more piece of evidence about the improved accuracy of modern-day blood glucose meters, these measurements were performed "by clinical personnel well-trained to the limitations of the test systems, the manufacturer's labelling, the safety practices, and the study protocol." A better protocol would have been to have the identical study performed by patients of all ages and hospital nurses involved with both subcutaneous and intravenous insulin dosing.

\section{Author Disclosure Statement}

S.G. has no competing financial interests. I. B. H. is a consultant for Roche and Abbott and conducts research for NOVO. 